\title{
L'adaptation aux évolutions récentes des vins vue par des vignerons d'AOP. Enquête en Anjou et Alsace (France)
}

\author{
Geneviève Teil ${ }^{*}$ \\ UMR SADAPT, INRAe, AgroParisTech, Université Paris-Saclay, 75005 Paris, France
}

\begin{abstract}
Résumé - La viticulture en appellation d'origine protégée (AOP) est considérée d'autant plus sensible au changement climatique qu'elle s'impose des contraintes réglementaires de localisation, d'approvisionnement, de pratiques et de qualité des vins. Ces obligations vouent-elles les AOP à une disparition rapide faute de pouvoir s'adapter aux changements à venir? Bien que l'anticipation de long terme du changement climatique ne soit pas vraiment à l'ordre du jour, les vignerons adaptent tous en continu leurs pratiques aux variations des conditions atmosphériques. Une enquête par entretiens auprès de vignerons angevins et alsaciens montre néanmoins qu'ils divergent dans la conception et la mise en œuvre de cette adaptation, notamment au moment de distinguer ce qui fait l'identité d'AOP à maintenir et les ressources acceptables pour y parvenir. De plus, cette enquête pointe l'émergence d'une façon originale de penser l'adaptation qui délègue à la vigne la charge de la bonne adaptation, défiant ainsi les plus sombres pronostics. Pour y parvenir, les producteurs expérimentent et renouvellent leurs pratiques vitivinicoles afin de rendre leurs vignes «résistantes» au changement et qu'elles continuent d'exprimer leur terroir et de manifester les qualités de leurs AOP.
\end{abstract}

Mots clés : changement climatique / qualité / adaptation / vin / AOP / terroir

\begin{abstract}
Adaptation to the recent changes in wines from PDO vine-growers' and wine-makers' point of view. A survey in Anjou and Alsace, France. Viticulture in Protected Designation of Origin (PDO) is considered all the more sensitive to climate change as it imposes regulatory constraints on location, supply, practices and quality of wines. Do these obligations doom PDOs to disappear quickly because they cannot adapt to the changes to come? Although long-term anticipation of climate change is not really on their agenda, winegrowers are all continuously adapting their practices to variations in weather conditions. A survey by interviews with Angevin and Alsatian winegrowers nevertheless shows that they diverge in the design and implementation of this adaptation, especially when it comes to distinguishing what makes the identity of PDO to be maintained and the acceptable resources to achieve it. In addition, this survey points to the emergence of an original way of thinking about adaptation which delegates the burden of good adaptation to the vine, thus defying the darkest prognoses. To achieve this, producers are experimenting and renewing their vine-growing practices in order to make their vines "resistant" to change so that they continue to express their terroir and demonstrate the qualities of their PDOs.
\end{abstract}

Keywords: climate change / wine / quality / adaptation / PDO / terroir

\section{Introduction}

Parmi les acteurs économiques, les agriculteurs et tout particulièrement les viticulteurs et les vignerons sont en première ligne face au changement climatique : leur activité dépend en effet très directement des conditions atmosphériques locales, notamment des apports en lumière, en eau et en

\footnotetext{
* Auteur de correspondance :

genevieve.teil@agroparistech.fr
}

$\mathrm{CO}_{2}$, mais aussi d'événements ponctuels destructeurs - gel, grêle, sécheresse ou tempête. Des auteurs pressent donc les acteurs du monde agricole de s'adapter au changement climatique, autrement dit de prendre en compte cette tendance toujours renforcée au réchauffement et à l'augmentation de l'aléa climatique (Howden et al., 2007 ; Kiem et Austin, 2013; Rickards et Howden, 2012). Outre les questions générales soulevées par les politiques d'adaptation, l'agriculture pose en effet des problèmes spécifiques, dus à son lien immédiat avec les conditions atmosphériques, et très divers selon les cultures 
(Bryant et al., 1997; de Noblet et al., 2014; Dunlap, 2010 ; Howden et al., 2010; Iglesias et al., 2012; Rickards et Howden, 2012).

La vigne, une production relativement résistante à la sécheresse, emblématique et source de forte valeur ajoutée, a fait l'objet de travaux ciblés qui procèdent à un inventaire des ajustements techniques (van Leeuwen et al., 2019), réglementaires (White et al., 2009), variétaux (Duchêne et al., 2010), géographiques (Moisselin et al., 2002), mais aussi économiques (Howden et al., 2007) à envisager.

La production vitivinicole en zone d'appellation d'origine protégée (AOP) justifie une attention toute particulière, car la production y est contrainte par les cahiers des charges de zonage et de pratiques vitivinicoles admissibles; de plus, la qualité des vins doit y satisfaire à des normes particulières, notamment de typicité. Plusieurs auteurs se sont penchés sur les limitations imposées par les AOP à l'adaptation au changement climatique ; ils ont souvent auguré leur disparition à moins d'un assouplissement drastique de leurs contraintes: des changements d'encépagement (Jones et Webb, 2010 ; Shaw, 2012), un abandon au moins partiel de l'origine des raisins (Hayes et Battaglene, 2006; Mosedale et al., 2016) ou à défaut leur délocalisation et la migration vers des climats plus frais (Hannah et al., 2013; Moriondo et al., 2013 ; van Leeuwen et Darriet, 2016; White et al., 2009).

Ces prévisions alarmantes contrastent avec le peu de préoccupation manifesté par les vignerons envers le changement climatique dont ils attribuent les effets au changement technique (Teil, 2020), même si l'on note une possible évolution ces dernières années dans les vignobles du Sud. Leur indifférence mettrait-elle en danger l'avenir des appellations viticoles?

Cet article propose d'aborder cette question en focalisant l'analyse sur les entretiens avec les vignerons rencontrés lors de l'enquête sociotechnique de terrain dans les deux appellations d'origine de l'Anjou et plus particulièrement l'appellation Savennières, et de l'Alsace. Quoique septentrionaux, ces deux vignobles ont une pluviométrie faible, similaire en quantité totale au Languedoc, et dont l'aridité pourrait s'accentuer (Teil, 2020).

\section{Une enquête sociotechnique de terrain}

Pourquoi interroger des vignerons sur le changement climatique s'ils ne s'en préoccupent guère?

Certains des vignerons de notre enquête sont clairement engagés dans l'atténuation du changement climatique en cherchant, par exemple, à limiter leur usage des tracteurs. Mais rares sont ceux qui expérimentent des cépages ou des portegreffe adaptés à des températures en hausse par exemple, ou qui intègrent le changement climatique dans la conduite et la gestion à long terme de leurs vignobles. En revanche, ils s'adaptent tout au long du cycle phénologique de la vigne aux conditions atmosphériques variables dont dépendent les quantités et qualités finales de leur production; ils les anticipent, en essuient les effets quand ils ne parviennent pas à les atténuer, privilégiant les actions réversibles, mais prenant parfois aussi des décisions qui engagent à plus long terme l'avenir de leurs entreprises. Ils prennent des risques, se trompent parfois, et accroissent leur expérience au fil des millésimes.
La notion d'appellation est étroitement liée à celle de terroir, c'est-à-dire notamment l'environnement physique naturel, pédologique et surtout climatique du vignoble dont dépend la qualité des vins et que les vignerons ne peuvent modifier en profondeur : ils n'ont pas le droit de bâcher leurs cultures, par exemple, pour tenter de réguler les apports en eau et optimiser ainsi leur production; sauf dérogations, l'irrigation de la vigne est toujours interdite dans les appellations vinicoles françaises. Ils subissent donc des conditions atmosphériques qu'ils ne peuvent modifier. Les modes d'ajustement mis en œuvre sont ainsi plutôt des façons d'anticiper ou de retarder, de réorienter ou de compenser les effets indésirables d'un temps toujours incertain sur le développement de leurs vignes. Ils adaptent donc, mais au jour le jour, et avec une gamme de ressources réduite, les pratiques de conduite de leurs vignes, et en jouant, lorsque c'est possible, entre les expositions de leurs différentes parcelles. Leur horizon temporel est cadré par le cycle annuel de la vigne, et dans une moindre mesure par le cycle de vie assez long de cette plante pérenne. La question de l'adaptation des vignerons a donc bien un sens et cet article tente de rendre compte des questions qu'elle soulève.

Les enquêtes déjà disponibles ne sont pas très nombreuses ; une étude de Neethling et al. (2017) procède à un relevé très détaillé des différentes techniques viticoles mises en œuvre par des vignerons en Anjou. Limitée à la viticulture, elle n'aborde pas les questions liées à la typicité des vins ou associées aux arbitrages entre qualité et quantité. Nicholas et Durham (2012) détaillent les stratégies d'adaptation à court terme à une large gamme de stress environnementaux, mais en Californie où les pratiques et la qualité des vins ne sont donc pas soumis aux contraintes propres aux AOP. La qualité d'AOP constitue au contraire un point central pour l'analyse d'Aspøy (2019), qui rend compte des vins de Moselle, une situation assez similaire, semble-t-il, à celle des deux vignobles de l'étude. Cependant, l'analyse, centrée sur les évolutions de style des vins, n'aborde pas la question des pratiques, causes ou corrections de ces variations de style, au contraire au cœur de ce travail.

\section{Méthodologie d'analyse}

Cet article s'appuie sur un sous-ensemble des entretiens réalisés dans le cadre de l'étude LACCAVE, un programme de 4 ans (2012-2016) financé par le métaprogramme ACCAF (Adaptation au changement climatique de l'agriculture et de la forêt) de l'INRA. Les entretiens ont été conduits auprès de 26 vignerons et œnologues en Alsace et 22 en Anjou. Les enjeux méthodologiques sont exposés dans l'article de Teil (2020); nous n'en présentons ici que les grands traits et les éléments concernant plus particulièrement le sujet de cet article.

Le choix d'une enquête qualitative de terrain présente un double avantage. D'une part, il permet de rendre compte de l'adaptation en acte. Ensuite, comme tout cas de terrain ciblé sur une activité précise, il met en lumière la complexité multidimensionnelle des contraintes, choix et arbitrages réalisés dans le cours de l'action (Bryant et Garnham, 2013; Tarleton et Ramsey, 2008). Il fait ainsi ressortir la difficile mais cruciale question de la qualité des vins (Bernetti et al., 2012) et sa double contrainte sur le résultat et les pratiques de production. 
L'enquête par entretiens longs permet d'aller au-delà de l'enregistrement des pratiques en interrogeant systématiquement leur enchâssement selon les cas, dans des stratégies de production, des politiques commerciales, des raisonnements économiques, des anticipations de conditions atmosphériques, etc. Nous avons été particulièrement attentifs à ne faire aucune impasse sur les débats touchant à la qualité gustative des raisins ou des vins, un sujet crucial au cœur des débats sur l'adaptation, tout particulièrement dans les appellations d'origine.

L'exposé s'appuie sur une analyse de contenu des entretiens; comme toute analyse sociotechnique, elle est parcourue de considérations et arguments «techniques», qui orientent la réflexion des vignerons. Pour ne pas décourager le lecteur non spécialiste, nous avons intégré de façon aussi simplifiée et claire que possible ces savoirs vitivinicoles souples ou rigides, parfois controversés. Le lecteur spécialiste pourra être tenté d'en faire la critique. Mais ceci n'est pas un article d'agronomie viticole: ce n'est pas l'efficacité des pratiques qui nous intéresse, mais bien la façon dont les vignerons pensent l'adaptation en lien avec leur activité de production et de commercialisation dans le cadre des contraintes propres aux AOP.

Les personnes interrogées sont largement citées. Pour préserver leur anonymat, leur nom est codé selon les catégories d'activité, ici « $\mathrm{V} »$ pour vigneron, la région et un numéro arbitraire d'entretien. "VPdL10» est un vigneron d'Anjou.

L'article est structuré en deux sections. La première part de deux évolutions récentes des vins, la dispersion de la teneur en sucre des vins et les pratiques de désacidification, qui illustrent des divergences d'arbitrage entre ce qui fait l'identité d'un vin et ce qui constitue des ressources acceptables permettant de pérenniser cette identité. La seconde section développe plus généralement cette opposition qui amène les vignerons à penser la «résistance» de leurs vignes, renouvelant ainsi les pratiques d'adaptation à différents changements pouvant survenir dans les AOP.

\section{L'identité d'AOP face aux évolutions récentes des vins}

Les raisins et les vins changent, le constat est unanime (Teil, 2020). Les conditions climatiques sont cependant loin d'être la seule variable explicative pour les vignerons qui pointent plutôt d'autres changements dans les techniques, les projets d'entreprise, ou encore les prix et les consommateurs : «La plupart des gens ont adapté le produit au goût des gens. Et tout le monde sait que les gens apprécient de moins en moins les extrêmes, les amers, les acides, et ils veulent de plus en plus de salé et de sucré», VAl10.

Quelle qu'en soit la «cause» climatique, technique ou commerciale, la question des changements observés dans les vins est un sujet assez conflictuel dans les deux appellations de l'enquête, car il touche à l'identité gustative des vins, une notion encadrée par les règlements d'appellation, mais interprétée d'assez diverses façons selon les vignerons.

Les tensions sont particulièrement vives au sujet de la hausse des sucres des raisins et de la baisse de l'acidité des vins, deux évolutions récentes du goût des vins qui touchent très inégalement les vins selon les millésimes. Elles génèrent d'importantes disputes sur l'ajustement requis. Certains craignent dès lors un brouillage de "l'identité» des vins et insistent sur le désarroi du consommateur en supermarché qui ne sait plus, lorsqu'il achète un vin d'Alsace, s'il sera sec ou doux. Ils exigent donc une grande discipline collective de la part des producteurs de l'appellation pour qu'ils respectent la «typicité» des vins, entendue comme identité gustative. Mais cette analyse n'est pas toujours partagée.

\subsection{Plus de sucre}

Depuis Joseph Capus et son décret-loi de 1935, les appellations vinicoles portent une double différenciation qualitative : elles doivent distinguer des vins à la fois typiques, c'est-à-dire différents des autres, mais aussi les meilleurs possibles, faute de quoi elles ne sauraient résister à la pression concurrentielle, du fait de leurs contraintes et coûts de production. Cette recherche de qualité supérieure s'est traduite, tout particulièrement dans le vignoble alsacien, par une importante mais néanmoins inégale baisse des rendements.

Chez les vignerons qui cherchent plutôt des marchés de volume assortis d'une bonne maitrise du prix de revient, et donc plutôt des rendements plus élevés, l'élévation des taux de sucre est nette, mais elle permet d'abord de réduire le recours à la chaptalisation, un ajout de sucre autorisé par les cahiers des charges pour garantir un taux alcoolique minimum.

«Il y a 30 ans, les gens en général enrichissaient à 2,5 parce qu'ils avaient droit à 2,5 , ils ne se posaient pas trop de questions. Parfois, il y avait de l'enrichissement inutile. Aujourd'hui, je crois, qu'en moyenne, on enrichit autour de 0,5 à 0,8 , selon le millésime. Donc là aussi, on s'est rapproché de la réalité naturelle. Ça aussi, ça participe à l'évolution finalement constatée du vignoble», VAl7.

Ceux qui recherchent plutôt une différenciation en qualité supérieure, grâce en particulier à des rendements plus faibles, se réjouissent de voir les vins gagner en «concentration» et de reléguer une pratique de correction qu'ils jugent contraire au respect du terroir: "la vigne n'a jamais fait de sucre de betterave...! !.

Les baisses de rendement plus marquées qu'ils pratiquent apportent également un surcroît d'arômes, mais aussi de sucres dans les raisins et donc d'alcool dans les vins, qu'ils ne cherchent pas à corriger : en effet, de leur point de vue, il est la conséquence d'une expression plus concentrée, et donc meilleure, du terroir des vins. Ces vignerons cherchent alors de nouveaux équilibres, en jouant notamment avec l'acidité des vins:

«Aujourd'hui, j'ai un pressoir pneumatique, qui n'abîme pas du tout les peaux. [...Je ramasse] peut-être des raisins qui ont un petit peu de végétal encore dedans, mais que je ne vais pas extraire. Donc, je peux avoir des raisins peut-être un peu moins mûrs, qui vont garder de l'acidité. Ces dernières années, mon degré d'alcool a augmenté, mais le pH est descendu, dans les vins. Avant, j'étais à $13,5^{\circ}$ et j'avais des $p H$ de 3,40. Aujourd'hui, je suis à $14^{\circ}$ d'alcool, j'ai des $p H$ de 3 à 3,10. Donc, c'est peut-être antinomique avec le réchauffement climatique, mais ça veut dire que mes pratiques culturales ont influencé le $p H$ des vins aussi », VPdL17.

Cependant, dans les millésimes favorables, les taux de sucre sont tels que les fermentations laissent de plus en plus 
Tableau 1. Échelle française de sucrosité des vins.

Table 1. Wine sweetness scale of the European regulation.

\begin{tabular}{ll}
\hline Dénomination & Taux de sucre \\
\hline Sec & $4 \mathrm{~g} / 1$ de sucre au maximum \\
Demi-sec & Entre $4 \mathrm{~g} / 1 \mathrm{et} 12 \mathrm{~g} / 1$ \\
Moelleux (ou demi-doux) & Plus de $12 \mathrm{~g} / 1 \mathrm{et}$ atteint au maximum $45 \mathrm{~g} / 1$ \\
Liquoreux (ou doux) & Teneur minimale en sucre de $45 \mathrm{~g} / \mathrm{l}$, sans limite supérieure \\
\hline
\end{tabular}

souvent des sucres «résiduels» dans les vins. Plutôt que d'accepter de remonter leurs rendements, et ainsi de «diluer» leurs vins et leur expression de terroir, les vignerons proposent, pour lever l'embarras des acheteurs, de recourir à des informations aux consommateurs.

Il existe en France un ensemble de termes - sec, demi-sec, moelleux, liquoreux - qui permettent d'indiquer la quantité de sucres résiduels du vin (Tab. 1). En Anjou, les vignerons rejettent la catégorie «demi-sec », qu'ils considèrent dévalorisante : ni sec ni doux, elle ne parle pas aux consommateurs. En Alsace, on reproche à cette classification d'être trop analytique et de ne pas tenir compte de la sensation en bouche qui dépend aussi de l'équilibre sucre-acide et de la «concentration» des vins. La sensation sucrée est pour eux d'autant plus forte que les vins sont dilués et manquent d'équilibre.

Des vignerons alsaciens ont ainsi travaillé à la mise au point de diverses échelles de «sucrosité» des vins. Parmi elles, l'indice d'expression, appelé aussi Indice de sucrosité des Grands Vins d'Alsace, tente de se rapprocher de la perception gustative (Tab. 2). Cette échelle s'appuie sur une interprétation plus globale de la perception sucrée en bouche tenant compte de l'acidité, mais aussi de son évolution avec le vieillissement des vins. Elle ne comprend plus 4, mais 5 degrés et l'on peut avoir jusqu'à $6 \mathrm{~g} / \mathrm{l}$ de sucres résiduels, au lieu de 4 dans la précédente échelle, voire $9 \mathrm{~g} / \mathrm{l}$ si le vin a une forte acidité. Les deux premières catégories élargissent la notion de vin « $\mathrm{sec}$ » au lieu de la réduire comme le souhaitent les tenants d'une typicité resserrée. Pour les concepteurs de l'indice, le goût des vins doit être le résultat de l'expression de leur terroir, concentrée sur les petits rendements et les pratiques plus respectueuses de la vigne et des raisins, sans concessions envers les modes changeantes de la demande. Ils se retournent ainsi contre les vignerons partisans de la typicité «vive et fraîche», dénonçant leur recours à la chaptalisation et à la désacidification chimique. Ces techniques de correction gustative des vins sont de leur point de vue d'autant moins acceptables qu'elles sont rendues nécessaires par des rendements excessifs et des stratégies de production à bas coût qui dévoient l'expression de terroir des vins et dévalorisent l'appellation.

Sur ce sujet de l'augmentation des sucres dans les vins, la notion d'adaptation génère donc des arbitrages très différents, voire opposés, sur le partage à faire entre ce qu'il importe de conserver et ce qui constitue des ressources appropriées pour y parvenir. L'AOP est d'un côté une garantie de résultat, une qualité gustative étroitement définie qui requiert donc un éventail large de pratiques permettant de garantir cette qualité millésime après millésime. De l'autre, c'est une garantie plus orientée sur les moyens, un ensemble de pratiques qualitatives favorisant l'expression du terroir y compris ses variations annuelles, ce qui oblige à une certaine ouverture de la notion de typicité gustative des vins.

\subsection{Désacidifications}

Plutôt qu'un encadrement plus étroit des rendements, avec des seuils minimaux et maximaux par exemple, les échelles de sucrosité tentent de remettre au goût du jour une solution de longue date pour encadrer l'identité des vins : la déclinaison de l'identité selon une variété de styles de sucrosité au sein de l'appellation. Une telle option n'existe pas pour l'acidité.

Dans les vignobles de l'étude, tous deux septentrionaux, des producteurs défendent la nécessité de désacidifier dans les millésimes moins ensoleillés. Ils peuvent recourir à diverses techniques, dont la fermentation malolactique, au cours de laquelle des bactéries présentes dans le vin transforment l'acide malique en un acide moins fort, l'acide lactique. Cette fermentation abaisse donc l'acidité, générant ce qui est décrit comme un «assouplissement» des vins. Cette fermentation est connue de longue date; mais l'identification récente des bactéries lactiques du vin qui transforment l'acide malique en lactique en a fait une ressource technique. Toujours recherchée dans les vins rouges, son usage est très controversé dans les blancs, en Anjou comme en Alsace:

"Quand on a pris les vignes à Savennières en 89, on voulait faire un vin à la bourguignonne, on a fait les fermentations malolactiques, et on a passé ça en bois. Parce qu'on sortait de l'école, et on voulait faire autre chose. [...] On est vraiment revenu en marche arrière de tout le monde. Aujourd'hui, je ne veux surtout pas de malo [surnom familier de la fermentation malolactique] sur mes Savennières, parce que dans l'export... [...] Aujourd'hui, la différenciation, c'est la fraîcheur et cet équilibre entre la matière et l'acidité», VPdL21.

En Anjou, on lui reproche de donner un caractère bourguignon, avec des vins trop «mous» et «lactés», et, en Alsace, de détruire les notes aromatiques variétales des cépages alsaciens. Les partisans de la typicité vive et fraîche des vins lui préfèrent donc la désacidification chimique, une technique plus contrôlable et plus abordable que la gestion de l'acidité par la viticulture, mais qui nécessite d'inhiber le démarrage de la fermentation malolactique en ajoutant du $\mathrm{SO}_{2}$, antibactérien, au moût. Cet ajout est rejeté par les partisans de vins plus naturels et moins «fabriqués», qui veulent diminuer l'usage du soufre, en particulier pour bloquer cette fermentation. 
Tableau 2. Indice d'expression (indice de sucrosité des Grands Vins d'Alsace).

Table 2. Expression index.

\begin{tabular}{|c|c|c|c|}
\hline 1 & $\mathrm{Sec}$ & Vin techniquement sec ou se goûtant sec & $\begin{array}{l}0 \text { à } 6 \mathrm{~g} / 1 \text { de sucres résiduels et jusqu'à } 9 \mathrm{~g} / 1 \text { si l'acidité } \\
\text { totale est de } 7 \mathrm{~g} \text { d'acide tartrique/l minimum }(-2 \mathrm{~g} / 1 \text { par } \\
\text { rapport à la teneur en sucres résiduels })\end{array}$ \\
\hline 2 & D'esprit sec & $\begin{array}{l}\text { Vin n'étant pas techniquement sec, mais dont les } \\
\text { sucres ne se goûtent que très peu au palais et } \\
\text { s'intégreront lors de la maturation en bouteille }\end{array}$ & $\begin{array}{l}7 \text { à } 15 \mathrm{~g} / 1 \text { de sucres résiduels, mais toujours en fonction de } \\
\text { l'équilibre du vin (acidité, } \mathrm{pH} \text {, alcool, structure...) }\end{array}$ \\
\hline 3 & Demi-sec & $\begin{array}{l}\text { Vin avec une sucrosité moyenne qui se ressent } \\
\text { davantage dans la jeunesse du vin et qui } \\
\text { s'estompera progressivement avec la maturation en } \\
\text { bouteille }\end{array}$ & $\begin{array}{l}15 \text { à } 30 \mathrm{~g} / 1 \text { de sucres résiduels, mais toujours en fonction } \\
\text { de l'équilibre du vin (acidité, } \mathrm{pH} \text {, alcool, structure...) }\end{array}$ \\
\hline 4 & Moelleux & Vin avec une sucrosité présente à la dégustation & $\begin{array}{l}30 \text { à } 50 \mathrm{~g} / 1 \text { de sucres résiduels, qui restent perceptibles en } \\
\text { bouche également lors de la maturation en bouteille }\end{array}$ \\
\hline 5 & Doux & $\begin{array}{l}\text { Vin avec une sucrosité marquée (comme les } \\
\text { vendanges tardives ou les sélections de grains } \\
\text { nobles) }\end{array}$ & $50 \mathrm{~g} / 1 \mathrm{de}$ sucres résiduels et plus \\
\hline
\end{tabular}

«Malo ou pas malo sur le chenin? Ça a été longtemps un sujet de conversation, mais vous n'avez pas idée! [...] En Bourgogne, on a discuté avec $X$, [...elle] a dit: "La malo, ce n'est pas un problème! En fait, si on a la bonne culture à la vigne, on ne se pose plus la question". Et en fait, depuis la canicule de 2003, dans nos caves, la malo se déclenche. Alors qu'avant, elle ne se déclenchait pas. On a changé les fûts, on a nettoyé... Ça n'a rien changé. Alors, donc, on ne se pose plus la question, il y a des fûts qui la font, des fûts qui ne la font pas. Ça fait comme ça veut [...], on n'essaie pas de la bloquer au soufre, ni quoi que ce soit», VPdL4.

Par l'entremise, ici, des agents fermentaires, la nature contribue, comme le vigneron, à la qualité finale des vins. Mais laisser faire la nature n'est pas synonyme de laisser-aller; le vigneron peut au contraire s'appuyer sur des savoir-faire pointus pour tenter de cadrer en amont les marges d'action de la nature, par le contrôle de la production d'acide malique par la vigne par exemple.

L'acidité des vins est l'objet d'un pilotage aux diverses ressources qui dépendent largement de la part que le vigneron souhaite laisser à la vigne dans la production de la qualité des vins. Les arbitrages sur les ressources admissibles sont donc intimement liés au statut de la vigne dans la production du vin : un processus biologique au service d'un vigneron ou une médiation essentielle de l'expression du terroir à accompagner et guider.

La sucrosité et l'acidité des vins font donc face à des exigences d'identité commerciale assez similaires. Cependant, certains vignerons s'opposent à un encadrement trop rigide de cette identité et proposent de s'en remettre à un arbitrage inattendu, celui du terroir. C'est à lui de « décider » de ce que le vin doit être à chaque nouveau millésime. Ce faisant, ils déplacent l'identité du vin d'une identité rigide de résultat de produit à celle plus flexible d'une œuvre d'auteur (Teil, 2014).

Ces deux interprétations de l'ajustement acceptable des pratiques aux changements en cours reposent sur deux points de vue opposés de l'identité des AOP: un engagement de moyens ou de résultat. Toutes deux structurent deux modes assez opposés de conduite de la vigne au fil de son développement annuel, autrement dit des conceptions de l'adaptation des pratiques de vitiviniculture à l'évolution des conditions atmosphériques.

\section{Corriger, accompagner, faire résister}

«Une bouteille de 61 et une bouteille de maintenant, vous avez toujours cette minéralité qui est là, dans la bouche. Ça, c'est le terroir. Le terroir, il ne bouge pas. Par contre, entre 61 et maintenant, on n'a pas du tout vendangé de la même façon. En 61, c'était du vin qui avait du sucre résiduel. Maintenant, on essaie de faire des vins secs, donc on ne ramasse pas du tout la même chose», VPdL12.

Ce vigneron a une vision très déterministe du terroir physique (la vigne dans un sol et un climat) : il recouvre un ensemble de causes ou de facteurs qui produisent des effets que l'on retrouve dans les vins. Cette cause terroir a un caractère nécessaire: de même qu'il est impossible d'éviter la gravité, tout vin produit dans un terroir a inévitablement, selon eux, le caractère que lui donne la conjonction particulière des facteurs de ce terroir. Le terroir n'a besoin ni de porte-parole, ni de canal d'expression. Il est présent ou subi quel que soit le travail du vigneron, du moment, ajoutent quelques-uns, que celui-ci respecte le cahier des charges des pratiques vitivinicoles de son AOP. La question de la qualité de terroir des vins ne pose ici guère de problème; c'est plutôt la commercialisation qui oriente le travail du vigneron, avec son chapelet de conséquences ou d'orientations relatives à l'équipement de l'exploitation, les choix de marketing ou de rentabilité de l'entreprise, notamment.

Comme l'ont montré les disputes sur les taux d'acide et de sucre des vins, cette interprétation du terroir est controversée : «Ce n'est pas parce qu'un vigneron suit à la lettre le cahier des charges de l'AOP que ses vins acquièrent par nécessité une qualité de terroir des vins!». Le vigneron joue lui aussi, ajoutent-ils, un rôle majeur dans l'avènement de la qualité de terroir finale qu'il peut brouiller, estomper, et même effacer; celle-ci doit donc être protégée de ses interventions indésira- 
bles. Selon qu'ils pensent que le terroir s'impose, ou que le vigneron doit s'obliger à en respecter l'expression, ils diffèrent dans l'interprétation de l'adaptation, autrement dit, dans le choix de l'objectif et des ressources permettant de produire année après année, des vins de terroir.

\subsection{Les quantités à la vigne, la qualité au chai}

Dans un premier cas, le terroir étant assuré, le vigneron peut se consacrer à un autre arbitrage essentiel pour assurer le chiffre d'affaires nécessaire à son entreprise: celui des quantités et des qualités.

«Mais nous, ce qui est important, c'est vrai que ce n'est pas poétique ce que je vais raconter, mais c'est la vérité des choses. [...] On a quand même un matériel super, un outil de travail performant. Pourquoi ça? Parce que les vignes, il faut qu'elles aillent au rendement. Pour moi, ça, c'est important », VPdL19.

Tenu par des engagements de prix contenus, ce vigneron hiérarchise ses objectifs. Au risque de la caricature, on peut dire qu'il subordonne la qualité aux quantités. Compte tenu des incertitudes qui planent sur ces quantités jusqu'à la vendange, le plus souvent, les qualités sont ajustées au chai, une fois les quantités assurées. Le vigneron ci-dessus se plaint ainsi sans surprise des dispositions du cahier des charges d'appellation qui limitent les rendements, mais surtout les pratiques de correction admissibles des vins, concentration, chaptalisation, acidification, mouillage... Les mesures de correction des moûts et des vins sont des ressources essentielles pour lui, parce qu'elles lui permettent de tenir ses engagements qualitatifs à un coût acceptable, une fois les quantités assurées.

Certains de ses partenaires d'appellation refusent cependant cette subordination de la qualité à la quantité : « la qualité, cela se fait à la vigne, et non au chai!»; les corrections sont pour eux le signe d'une absence de visée qualitative du producteur, qui menace la réputation et donc la pérennité des appellations. Mais depuis quelques années, des vignerons ne se contentent plus faire le choix inverse, à savoir subordonner les quantités à la qualité.

\subsection{Respecter le terroir tout au long de la production}

Certains vignerons reprochent à l'adaptation par correction d'induire un lissage et une «standardisation» artificielle de la qualité des vins d'AOP. Ils dénoncent ainsi la rigidité de la typicité des cahiers des charges : dès lors que la qualité est une véritable qualité de terroir, elle est nécessairement variable selon les millésimes et la multiplicité des «facettes » des terroirs.

Ces vignerons s'engagent dans une autre voie d'adaptation tendue par le « respect du terroir» et la nécessité de ménager la plus grande place possible à son vecteur, la vigne. À cet effet, ils cherchent à limiter, autant que faire se peut, les interventions du vigneron qui viendraient brouiller l'expression du terroir ou la corriger : le vigneron doit accompagner la vigne dans son environnement climato-pédologique, attentif à ces qualités particulières qu'elle produit à chaque nouveau millésime. Ce faisant, ces vignerons inventent une forme innovante de typicité de terroir, dont la souplesse permet d'intégrer les variations dues à l'aléa climatique, du moins ses variations ordinaires et non «atypiques».
Ces deux façons de penser l'adaptation et le travail du vigneron, «l'adaptation-correction » et « l'adaptation-accompagnement», rappellent deux modèles pédagogiques. Dans le premier, l'objectif de l'enseignement à atteindre est prédéfini - l'élève doit réussir un examen portant sur des questions ou domaines prédéfinis - et le professeur doit recourir à toutes les techniques propres à faire apprendre à l'élève ce qu'il doit savoir. Dans le second, l'accent est mis sur « l'éveil » de l'élève plus que sur l'objectif d'acquisition de connaissances; la pédagogie est opportuniste et s'appuie sur les intérêts particuliers que l'élève manifeste au cours de son développement.

Les deux pédagogies requièrent la même étroite observation du développement des enfants et diffèrent selon le rôle imparti à chacun dans son pilotage. De la même façon, les deux adaptations des vignerons privilégient soit la réussite d'un objectif préétabli, soit «l'épanouissement» de la vigne. Cette seconde façon de penser l'adaptation procède à un autre découpage entre ce qui doit être stabilisé et ce qui peut être une ressource à cet effet, qui nourrit les conflits au sein des AOP.

\subsection{Adaptation incrémentale versus transformationnelle?}

Les notions d'adaptation «incrémentale» et «transformationnelle» permettent à Rickards et Howden (2012) de différencier deux familles de pratiques d'adaptation. La première est ciblée, ajustée à des objectifs précis, induisant des transformations limitées et circonscrites. La seconde a des effets moins bien identifiables a priori, car elle génère des cascades d'interactions plus ou moins imprévisibles, rendant la transformation induite beaucoup plus complexe à saisir. Ils la rapprochent de ce fait de l'idée de coévolution (Wise et al., 2014).

On peut rapprocher les pratiques «d'adaptation-correction » de l'adaptation incrémentale, faite d'interventions aux effets prévisibles et cadrés, et «l'adaptation-accompagnement » d'une coévolution de la vigne et du vigneron, car dans ce dernier cas, les effets des pratiques d'adaptation, de la vigne en particulier, sont moins clairement prévisibles et objectivables, car produits dans le cours de l'action. Mais le point essentiel apporté par l'adaptation-accompagnement est ailleurs : l'abandon à la vigne de la maîtrise sur les adaptations à produire. Il n'appartient plus au vigneron de corriger ces raisins plus sucrés ou ces vins moins acides.

Cette quête d'un terroir authentique, c'est-à-dire exempt de «dérives» de quelque nature qu'elles soient, a amené ces dernières années un renouveau des pratiques vitivinicoles ressources de l'adaptation, qui se disent plus « respectueuses » des terroirs. Mais surtout, elle transforme l'interprétation du terroir physique: au lieu d'un fournisseur de matières premières sensible aux conditions atmosphériques, il devient un «partenaire » du vigneron qui décide de la qualité de terroir. Aujourd'hui, les vignerons préoccupés d'expression de terroir font un pas de plus: rendre la vigne résiliente.

\subsection{Rendre la vigne résiliente}

Une expression revient souvent chez les vignerons: ils veulent rendre leurs vignes « résistantes», se déchargeant sur elles de la tâche d'amortir ou de compenser les effets d'un 
excès ou d'un déficit hydrique par exemple. Ils proposent tout d'abord de mettre les plantes à l'abri des variations de surface, grâce à un enracinement profond dans le sous-sol où ces variations sont atténuées. Cet enracinement exige des techniques de culture adaptées avec, par exemple, le retour à de fortes densités de plantation, la préférence pour le labour plutôt que pour les désherbants et le rejet des fertilisants de surface qui favorisent un développement racinaire peu profond. Ensuite, ils tentent d'aider la vigne à «supporter» la variabilité des conditions atmosphériques en essayant de diminuer sa réactivité par la limitation des rendements, le renforcement de la biodiversité clonale et le retour à l'agroforesterie notamment. À côté de l'organisation de cette résistance "passive», ils s'efforcent de restaurer ou de multiplier les équilibres et interactions entre la vigne et son environnement, afin qu'ils jouent le rôle d'amortisseurs des variations et agressions.

En l'aidant à «résister», le vigneron veut renforcer la capacité de sa vigne à exprimer le terroir malgré l'augmentation de l'aléa climatique. Mais il contribue aussi à une plus grande résilience de ses vignes au changement climatique qui s'accentue.

Source d'un important renouvellement technique, les efforts des vignerons pour «retrouver» l'expression perdue de leur terroir ont tout d'abord amené un accroissement de la variabilité gustative des vins, à l'origine d'importants conflits au sein des AOP (Teil, 2011). Avec cette dernière approche qui privilégie aujourd'hui la résilience de la vigne, c'est au contraire sa capacité à ne pas réagir aux changements qui est recherchée :

«Mais moi, je ne trouve pas que j'aie une grande variabilité. Quand je vois mes rendements qui sont hyper stables... Il y a 2008 qui était un peu plus faible, mais autrement, à un ou deux hectos près, je suis toujours pareil. C'est étonnant! Si bien que je ne trouve pas que l'effet millésime soit évident. Je te dis, parce que je vendange quand j'ai décidé de vendanger! », VPdL10.

Ce travail de passivation des réactions de la vigne, joint à une décision de vendanger appuyée sur des critères qualitatifs explicites, pourrait conduire à limiter la variabilité des expressions de terroir. Il pourrait ainsi faciliter la coexistence devenue problématique entre les deux grandes démarches commerciales qui font ensemble le succès des AOP: la production de volumes de vins à la typicité prédéfinie et la recherche toujours relancée de la qualité.

\section{Conclusion}

Les vignerons de notre enquête n'inscrivent pas le changement climatique en tête de leurs préoccupations, mais ils s'adaptent néanmoins à de multiples changements qui ne cessent de survenir dans leur environnement et tout particulièrement les conditions atmosphériques qui influent fortement sur leur production.

Les pratiques vitivinicoles d'ajustement à court terme sont une ressource essentielle pour les vignerons; relativement réversibles, elles leur permettent de s'ajuster à un aléa climatique croissant. De plus, ces pratiques font l'objet d'une intense réflexion et d'expérimentations de la part des vignerons: à côté de la démarche classique de correction des effets des changements et de celle plus originale d'accompagnement, une autre démarche d'adaptation est en train de s'inventer, qui vise à limiter l'intensité des effets des changements environnementaux. La richesse des réflexions des acteurs sur la contribution de la vigne à la qualité des vins et les pratiques de conduite admissibles souligne l'importance des modes de conduite vitivinicoles dans les AOP, à côté du déplacement ou de l'allègement du zonage AOP et de l'innovation variétale.

Bien qu'en conflit, les conceptions de l'adaptation qui ressortent de cette étude réalisent ensemble ce qu'aucune ne parvient à faire seule: changer sans perdre son identité. Ce serait donc sans doute une erreur de vouloir trancher les désaccords ou d'ignorer ces modes d'adaptation alternatifs qui, outre le renouveau de la réflexion vitivinicole qu'ils induisent, peuvent contribuer à une plus grande résilience globale des appellations.

Notons cependant que les résultats de cette étude sont très largement liés aux débats sur la qualité de terroir qui a touché l'ensemble des appellations françaises depuis les années 1990. Cette spécificité encore assez française, qui place la notion de terroir au cœur des appellations d'origine, explique peut-être que ces discussions soient relativement absentes de l'enquête menée en Allemagne par Aspøy (2019), en Californie par Nicholas et Durham (2012) et marginales en Espagne par exemple.

Remerciements. Nos plus chaleureux remerciements vont à l'ensemble de l'équipe du programme LACCAVE qui a accueilli ces recherches.

\section{Références}

Aspøy H. 2019. The notion of Mosel wine and its controversies. British Food Journal 121(12): 3076-3088. DOI: 10.1108/BFJ10-2018-0718.

Bernetti I, Menghini S, Marinelli N, Sacchelli S, Sottini VA. 2012. Assessment of climate change impact on viticulture: economic evaluations and adaptation strategies analysis for the Tuscan wine sector. Wine Economics and Policy 1(1): 73-86. DOI: 10.1016/j. wep.2012.11.002.

Bryant CR, André P, Provençal D, Singh B, Thouez J-P, El Mayaar M. 1997. L'adaptation agricole aux changements climatiques : le cas du Québec. Le Climat 14(2): 81-97 (numéro spécial : Les défis des changements environnementaux à l'échelle planétaire).

Bryant L, Garnham B. 2013. Beyond discourses of drought: the micro-politics of the wine industry and farmer distress. Journal of Rural Studies 32: 1-9. DOI: 10.1016/j.jrurstud.2013.03.002.

de Noblet N, Levrault F, Meybeck A. 2014. Agriculture et changement climatique - Regards croisés sur l'agriculture et le changement climatique. Notes et études socio-économiques (38): 95-120.

Duchêne E, Huard F, Dumas V, Schneider C, Merdinoglu D. 2010. The challenge of adapting grapevine varieties to climate change. Climate Research 41(3): 193-204. DOI: 10.3354/cr00850

Dunlap RE. 2010. Climate change and rural sociology: broadening the research agenda. Rural Sociology 75(1): 17-27. DOI: 10.1111/ j.1549-0831.2009.00010.x.

Hannah L, Roehrdanzb PR, Ikegamib M, Shepardb AV, Shawc R, Tabord G, et al. 2013. Climate change, wine, and conservation. Proceedings of the National Academy of Science: 110(17): 69076912. DOI: $10.1073 /$ pnas.1210127110. 
Hayes P, Battaglene T. 2006. Regulatory response to climate change. Bulletin de l'OIV 79(909/910): 697-708.

Howden S, Crimp S, Nelson R. 2007. Adapting agriculture to climate change. Proceedings of the National Academy of Sciences 104: 19691-19696.

Howden S, Crimp S, Nelson R. 2010. Australian agriculture in a climate of change. In Jubb I, Holper P, Cai W, eds.Managing Climate Change: Papers from the Greenhouse 2009 Conference. Collingwood (Aus): CSIRO Publishing, pp. 101-112.

Howden SM, Soussana J-F, Tubiello FN, Chhetri N, Dunlop M, Meinke H. 2007. Adapting agriculture to climate change. Proceedings of the National Academy of Sciences 104(50): 19691. DOI: 10.1073/pnas.0701890104.

Iglesias A, Quiroga S, Moneo M, Garrote L. 2012. From climate change impacts to the development of adaptation strategies: challenges for agriculture in Europe. Climatic Change 112(1): 143168. DOI: 10.1007/s10584-011-0344-x.

Jones GV, Webb LB. 2010. Climate change, viticulture, and wine: challenges and opportunities. Journal of Wine Research 21(2-3): 103-106. DOI: 10.1080/09571264.2010.530091.

Kiem A, Austin E. 2013. Drought and the future of rural communities: opportunities and challenges for climate change adaptation in regional Victoria, Australia. Global Environmental Change 23: 1307-1316. DOI: 10.1016/j.gloenvcha.2013.06.003.

Moisselin JM, Schneider M, Canellas C, Mestre O. 2002. Les changements climatiques en France au $\mathrm{xx}^{\mathrm{e}}$ siècle : étude des longues séries homogénéisées de température et de précipitations. La Météorologie 38: 45-56. DOI: 10.4267/2042/36233.

Moriondo M, Jones GV, Bois B, Dibari C, Ferrise R, Trombi G, et al. 2013. Projected shifts of wine regions in response to climate change. Climatic Change 119(3): 825-839. DOI: 10.1007/ s10584-013-0739-y.

Mosedale JR, Abernethy KE, Smart R, Wilson RJ, Maclean IMD. 2016. Climate change impacts and adaptive strategies: lessons from the grapevine. Global Change Biology 22(11): 3814-3828. DOI: 10.1111/gcb.13406.

Neethling E, Petitjean T, Quenol H, Barbeau G. 2017. Assessing local climate vulnerability and winegrowers' adaptive processes in the context of climate change. Mitigation and Adaptation Strategies for Global Change 22(5): 777-803. DOI: 10.1007/s11027-015-9698-0.
Nicholas KA, Durham WH. 2012. Farm-scale adaptation and vulnerability to environmental stresses: insights from winegrowing in Northern California. Global Environmental Change-Human and Policy Dimensions 22(2): 483-494. DOI: 10.1016/j.gloenv cha.2012.01.001.

Rickards L, Howden SM. 2012. Transformational adaptation: agriculture and climate change. Crop and Pasture Science 63(3): 240-250. DOI: 10.1071/CP11172.

Shaw TB. 2012. A climatic analysis of wine regions growing pinot noir. Journal of Wine Research 23(3): 203-228. DOI: 10.1080/ 09571264.2012.697356.

Tarleton M, Ramsey D. 2008. Farm-level adaptation to multiple risks: climate change and other concerns. Journal of Rural and Community Development 3: 47-63.

Teil G. 2011. Quand les acteurs se mêlent d'ontologie. Revue d'anthropologie des connaissances 5(2): 437-462. DOI: 10.3917/ rac.013.0437.

Teil G. 2014. Nature, the co-author of its products? An analysis of the recent controversy over "rejected" AOC wines in France. Journal of World Intellectual Property 17(3-4): 96-113. DOI: 10.1002/ jwip. 12022.

Teil G. 2020. Les vignerons seraient-ils climato-sceptiques ? Les effets du changement climatique analysés par les vignerons dans les appellations d'origine protégée d'Anjou et d'Alsace. Cahiers agricultures 29. DOI: 10.1051/cagri/2019030.

van Leeuwen C, Darriet P. 2016. The impact of climate change on viticulture and wine quality. Journal of Wine Economics 11(1): 150-167. DOI: 10.1017/jwe.2015.21.

van Leeuwen C, Destrac-Irvine A, Dubernet M, Duchene E, Gowdy M, Marguerit E, et al. 2019. An update on the impact of climate change in viticulture and potential adaptations. Agronomy-Basel 9 (9): 20. DOI: 10.3390/agronomy9090514.

White MA, Whalen P, Jones GV. 2009. Land and wine. Nature Geoscience 2: 82. DOI: 10.1038/ngeo429.

Wise RM, Fazey I, Stafford Smith M, Park SE, Eakin HC, Archer Van Garderen ERM, et al. 2014. Reconceptualising adaptation to climate change as part of pathways of change and response. Global Environmental Change 28: 325-336. DOI: 10.1016/j.gloenv cha.2013.12.002.

Citation de l'article : Teil G. 2020. L'adaptation aux évolutions récentes des vins vue par des vignerons d'AOP. Enquête en Anjou et Alsace (France). Cah. Agric. 29: 8. 